\section{Reproductive disease in birds}

\section{Yvonne van Zeeland}

Reproductive disease is commonly seen in parrots, with female birds generally being considered more prone to developing reproductive disease than males. Disease in the female is often associated with the complex process of egg laying and may include egg retention (i.e. delayed oviposition), dystocia (i.e. inability to pass a formed egg), chronic egg laying, oviductal prolapse and egg-related peritonitis.

Dystocia, also referred to as egg-binding, is a relatively common and potentially life-threatening condition for which various different causes have been defined. Of these, oviductal dysfunction due to hypocalcaemia is considered the most important. Birds with dystocia frequently present with straining and secondary prolapse of the cloaca and/or oviduct. In case of a prolapsed oviduct it is important to first remove the egg, following which it may be decided whether to reposition or remove the oviduct.

In the absence of a prolapse, prostaglandin E may be applied topically on the uterovaginal sphincter, resulting in a dilation of the sphincter and initiation of uterine contractions which aid in oviposition. Oxytocin will also stimulate these contractions, but should be used with caution as its use is contraindicated when the uterovaginal sphincter is closed.

Egg-related peritonitis may also result as a sequela to egg-binding, often recognisable by the presence of ascites, whereby large amounts of proteinaceous material can be found upon cytological examination of the fluid. Following stabilisation of the patient, surgery may be performed to remove the affected oviduct and egg debris. Prognosis remains reserved, however.

Chronic egg laying may sometimes be an underlying factor in the pathogenesis of the above-mentioned reproductive diseases. Particularly cockatiels and lovebirds are notorious egg layers, which will often continue to lay eggs as long as the laid ones keep on being removed from the nest. Eliminating the factors that trigger egg laying (e.g. long daylight, nest boxes, nesting materials, sprouted seeds, fruits) may help in some cases. In various patients, management changes will not suffice, thereby warranting use of hormonal or surgical intervention. In the past, medroxyprogesterone was commonly used, but was found to have many side effects. With the arrival of newer, equally effective but safer drugs, such as long-acting gonadotropin-realeasing hormone $(\mathrm{GnRH})$ analogues, its use is currently considered obsolete.

Aside from problems associated with egg laying, neoplastic conditions may also affect the gonads. The most important of these are the Sertoli cell tumours, which are commonly found in elderly male budgerigars. The most striking clinical feature in these patients is a change in cere colour, resulting from hyperoestrogenism. Other typical signs may include abdominal enlargement and unilateral paresis. Surgical excision may be attempted, but is often unsuccessful due to proximity of the large vessels, which increase the risk of fatal bleeding. Ovarian tumours have also been reported in parrots, most frequently budgerigars and cockatiels. Abdominal enlargement is a common finding, with surgical removal being equally challenging as in males. Recently, however, depot $\mathrm{GnRH}$ agonists have been found successful in decreasing the size of ovarian and testicular tumours, thereby providing a viable alternative to enhance the bird's quality of life.

\section{KEY LEARNING OBJECTIVES}

- Reproductive disease is more frequently seen in female parrots, compared to male parrots

- Appropriate nutrition plays a pivotal role in the prevention and treatment of many reproductive diseases

- The depot GnRH agonist deslorelin can successfully inhibit egg laying and may also be used in the prevention and treatment of reproductive disease in parrots

\section{MULTIPLE CHOICE QUESTIONS}

1. A budgerigar with brown ceres is presented to your practice with a distended abdomen and paresis of the left leg. Which of the following options does NOT fit within your differential diagnoses list?

a. A female budgerigar with a granulosa cell tumour

b. A female budgerigar with a theca cell tumour

c. A male budgerigar with a seminoma

d. A male budgerigar with a Sertoli cell tumour

2. What is the most common cause for egg binding in parrots?

a. Excessive egg laying as a result of continuous removal of laid eggs

b. Hypocalcaemia due to being fed a diet deficient in calcium and vitamin D

c. Laying of excessively large eggs in combination with a lack of dilation of the pelvis

d. Metaplasia of the oviductal mucosa due to dietary-related hypovitaminosis A

3. A 36-year-old grey parrot is presented to your practice with clinical signs of egg binding. Abdominal palpation reveals presence of an egg, which is found to be normally calcified on radiographs. No other abnormalities are present. Aside from supportive care, you decide to medically stimulate oviposition. Which of the following drugs should be considered first choice for inducing egg laying in an egg-bound bird?
a. Arginine vasotocin
b. Prostaglandin E
c. Prostaglandin $\mathrm{F} 2 \alpha$
d. Oxytocin

4. Deslorelin has been found useful to prevent reproductive disease in parrots. How does this hormone exerts its mode of action?
a. Suppression of $\mathrm{GnRH} /$ luteinising hormone releasing hormone $(\mathrm{LHRH})$ release
b. Stimulation of inhibin release
c. Suppression of luteinising hormone $(\mathrm{LH})$ release
d. Stimulation of progesterone release 


\section{Exotics}

5. Which of the following statements is correct with regard to excessive egg laying in parrots?

a. Surgical removal of the oviduct will guarantee cessation of ovarian activity and should therefore be considered as the preferred treatment option

b. Excessive egg laying is predominantly seen in psittacine species that belong to the group of indeterminate layers c. Shortening daylight (8L:14D) and removal of nest boxes and nesting materials will usually be sufficient to stop egg laying in parrots from equatorial regions

d. Medroxyprogesterone is considered a safe and effective treatment for parrots with excessive egg laying
Review of common diseases of ornamental fish

\section{Peter Scott}

The keeping or ornamental fish in aquaria and ponds is an extremely popular hobby with a long history. The supply of fish has evolved over the years and is extremely professional in handling of the fish involved, which often travel great distances around the world. The associated 'technology' for maintaining the fish has also developed considerably.

This session will briefly outline the numbers and supply routes of ornamental fish, the structure of the UK industry and the controls which must be in place to protect native populations from imported disease.

The various common diseases, viral, bacterial, protozoal, metazoan, fungal and non-infectious, which may be seen in pondfish and in aquaria will be illustrated and appropriate treatment options will be discussed. The pathogenesis of disease in the captive situation will be discussed and what measures can be taken to reduce problems. Although not 'common', notifiable disease will be covered.

\section{KEY LEARNING OBJECTIVES}

To gain an understanding of the pet fish industry To be aware of the common diseases of pet fish To be able to understand the problems seen in pet fish and contribute towards the solutions

\section{MULTIPLE CHOICE QUESTIONS}

1. What are the most commonly seen diseases of ornamental fish?
a. Viral
b. Bacterial
c. Protozoal
d. Endemic

2. Monogenean flukes are commonly found in which parts of the body?
a. Skin and gill
b. Gut
c. Kidney
d. Eyes

3. What is fishkeeper's finger or aquarist's arm caused by?
a. Mycobacteria sp
b. Erisipelothrix $\mathrm{sp}$
c. Streptococcus sp
d. Digenean trematodes

4. Which of these is not true?
a. Use of antibiotics via the water is bad practice
b. Malachite green has been banned for use in fish
c. Ich is the most important disease of captive fish worldwide
d. Fish are excluded from the Veterinary Surgeons Act

5. Which treatment aspect is not a common part of routine therapy of ulcers on individual koi?
a. Antibiotics by injection or food
b. Debridement and barrier products
c. Approx. $5 \mathrm{~g}$ of salt per litre in quarantine or the pond
d. Antibiotics in the water in quarantine or the pond

\section{Getting diagnostic samples and understanding water quality}

\section{Peter Scott}

There are two areas for testing in investigating fish health: these are the fish and their environment.
Fish can be anaesthetised, radiographed and submitted for computed tomography (CT) or magnetic resonance imaging (MRI) scanning.

Blood samples and biopsy samples can be taken, even laparoscopically. Postmortem investigation is routine and valuable: fish autolyse extremely rapidly so the use of remote laboratories is limited in this regard. Practitioners need to be aware of techniques and appropriate sampling.

Gill and skin scrapings are routine tasks examining for common parasites; these can be carried out on live fish, anaesthetised fish or indeed at postmortem. Histopathology and bacteriology can be very valuable if the correct samples are taken. Specialist virology is also available.

Captive fish are totally dependent on keepers to 